msh-mss Mathématiques et sciences humaines

176 | Hiver 2006

Varia

\title{
La logique probabiliste de Gabriel Cramer
}

Gabriel Cramer's probabilistic logic

\section{Thierry Martin}

\section{OpenEdition}

Journals

Édition électronique

URL : http://journals.openedition.org/msh/3647

DOI : $10.4000 /$ msh.3647

ISSN : 1950-6821

\section{Éditeur}

Centre d'analyse et de mathématique sociales de l'EHESS

\section{Édition imprimée}

Date de publication : 1 décembre 2006

Pagination : 43-60

ISSN : 0987-6936

\section{Référence électronique}

Thierry Martin, «La logique probabiliste de Gabriel Cramer ». Mathématiques et sciences humaines [En ligne], 176 | Hiver 2006, mis en ligne le 28 juillet 2006, consulté le 23 juillet 2020. URL : http:// journals.openedition.org/msh/3647 ; DOI : https://doi.org/10.4000/msh.3647 


\title{
LA LOGIQUE PROBABILISTE DE GABRIEL CRAMER
}

\author{
Thierry MARTIN ${ }^{1}$
}

\begin{abstract}
RÉSUMÉ - Dans les années 1745, l'algébriste Gabriel Cramer professa un Cours de logique, demeuré inédit jusqu'à nos jours et dont une partie importante est consacrée à la connaissance probable. Ce texte est la source de l'article "Probabilité » de l'Encyclopédie de Diderot et D'Alembert. Nous nous proposons ici de restituer la représentation de la logique probabiliste que ce texte développe, et nous montrons en quoi $: 1^{\circ}$ ) il constitue un bon témoin de l'état de développement de la pensée probabiliste dans la première moitié du XVIII siècle et des difficultés que rencontre sa formalisation, $2^{\circ}$ ) il manifeste une clarté et une rigueur que le résumé fourni par l'Encyclopédie n'a pas su respecter.

MOTS-CLÉS - Cramer, Encyclopédie, Logique probabiliste, Probabilité des témoignages

SUMMARY - Gabriel Cramer's probabilistic logic

Around 1745, the algebrist Gabriel Cramer gave a course of lectures on Logic, which was unpublished until to-day and of which an important part was dedicated to probable knowledge. The article "Probability" from the Diderot-D'Alembert's Encyclopaedia originates from these lectures. In this paper, we intend to disclose the representation of the probabilistic logic developed in this text, and we show in what way: $1^{\circ}$ ) this text highly testifies to the development of the probabilistic thought in the first half of the XVIIIth century and to the difficulties met by its formalization, $2^{\circ}$ ) it reveals a clearness and an exactness which the abstract given by the Encyclopaedia failed to account for.
\end{abstract}

KEY-WORDS - Cramer, Encyclopaedia, Probabilistic Logic, Probability of testimony

Le présent travail porte sur un texte inédit de Gabriel Cramer, à savoir le cours de logique qu'il professa à Genève, dans les années 1745, et dont une partie importante est consacrée à l'étude de la connaissance probable. Pourquoi prendre ce texte pour objet d'étude ? La question mérite d'être soulevée, car généralement l'historien des sciences dirige son intérêt vers des textes qui, par leur influence immédiate ou leur postérité, ont marqué l'histoire de la discipline. Or, ce n'est justement pas le cas de la Logique de Gabriel Cramer, puisque ce texte n'a jamais été publié. Il est vrai qu'il a donné naissance à plusieurs articles de l'Encyclopédie, et notamment à l'article Probabilité. Mais ce serait alors plutôt à cet article, à sa réception et aux débats qu'il a pu susciter, qu'il faudrait s'intéresser pour chercher à mettre au jour le rôle qu'il a pu jouer dans l'édification du calcul des probabilités. Le présent projet est pourtant légitime. Deux arguments suffiront à l'attester, l'un de portée générale, l'autre relatif à la personne même de Gabriel Cramer.

1. Pour comprendre comment se construit une discipline scientifique, il ne suffit pas d'en étudier les éléments moteurs, sources des innovations ou des transformations

\footnotetext{
${ }^{1}$ Laboratoire Logiques de l'Agir, Université de Franche-Comté, thierry.martin@univ-fcomte.fr
} 
qui s'y font jour. Il faut également considérer l'exposition de ses résultats, en quelque sorte solidifiés sous forme de savoir constitué, car ce sont justement ceux-ci qui, témoignant de ce que l'on peut penser ou édifier à tel moment de son développement, permettent de rendre compte de la possibilité des innovations et transformations du savoir.

2. Gabriel Cramer n'est pas un auteur quelconque, et ceci pour au moins deux raisons :

- D'une part, il s'est illustré par ses travaux d'algèbre, relatifs notamment aux systèmes d'équations linéaires. On peut donc s'attendre à trouver dans ce texte, sinon directement une application, du moins une illustration de la démarche algébrique.

- D'autre part, Gabriel Cramer est également à l'origine des travaux probabilistes de Buffon, et notamment de son analyse du problème de Saint-Pétersbourg et de la notion d'espérance morale, ce dont témoigne la correspondance échangée par Buffon et Cramer en octobre $1730^{2}$. Buffon reconnaît d'ailleurs sa dette à son égard au $\S \mathrm{XV}$ de l'Essai d'arithmétique morale, où il écrit à propos des mathématiques :

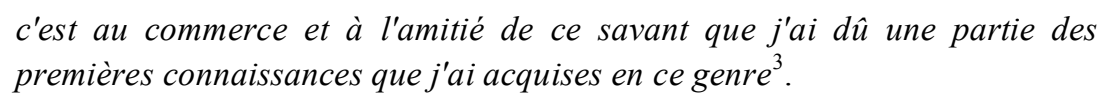

On peut donc faire l'hypothèse que ce texte, œuvre d'un mathématicien à la fois informé et compétent, propose une bonne présentation de la pensée probabiliste, telle qu'on pouvait la concevoir dans la première moitié du XVIII ${ }^{\mathrm{e}}$ siècle. Mais on ne peut espérer y trouver des développements mathématiques novateurs, son objet n'étant pas de rédiger un traité destiné aux mathématiciens eux-mêmes, ni même un cours à leur usage, mais de dispenser un enseignement aisément compréhensible par qui n'est pas familiarisé avec l'abstraction mathématique. Et de fait, on constate que Cramer prend soin de limiter au minimum le recours explicite aux mathématiques.

Il n'est évidemment pas possible de suivre ici en détail le développement de l'analyse de Cramer. Après avoir identifié notre document de travail, nous limiterons donc notre propos à illustrer la démarche qu'il en œuvre, en considérant plus particulièrement tout d'abord la progression du texte, ensuite la façon dont il met en place le concept de probabilité, enfin son analyse de la probabilité des témoignages.

\section{1. ÉTABLISSEMENT DU TEXTE}

\subsection{HISTORIQUE}

L'historique du texte a été retracé par Jean-Daniel Candaux dans l'article «Un auteur (et même deux) pour Idée, Induction, Probabilité: Monsieur de Lubières, encyclopédiste ${ }^{4}$. Jean-Daniel Candaux établit notamment que le Cours de Logique de Gabriel Cramer est la source dont fut tiré l'article Probabilité de l'Encyclopédie, article anonyme et antérieurement attribué à Diderot par Jean Mayer ${ }^{5}$.

La correspondance de Charles Bonnet avec Jean Senebier, bibliothécaire à Genève, autorise à admettre que les articles Idée, Induction et Probabilité de

\footnotetext{
${ }^{2}$ Cf. [Weil, 1961].

${ }^{3}$ [Buffon, 1777, p. 78]. Sur la relation de Buffon à Cramer, cf. également [Hanks, 1966, p. 17-61].

${ }^{4}$ [Candaux, 1993].

${ }^{5}$ [Mayer, 1991].
} 
1'Encyclopédie furent rédigés par Monsieur de Lubières ${ }^{6}$, celui-ci ayant, précise Charles Bonnet, «tiré de la Logique manuscrite de Mr Cramer» au moins les articles Idée et Probabilité.

Charles Bonnet donne quelques précisions sur le texte de Cramer. Il signale notamment dans ses Mémoires autobiographiques que «M. Cramer avait commencé à composer une Logique pour servir à l'instruction d'une jeune Dame d'un grand mérite ». Et il ajoute : «Il m'avait entretenu plus d'une fois de cet ouvrage; et je m'étais aperçu qu'il en était assez satisfait pour n'être pas éloigné de le publier... ${ }^{7}$. On peut en retenir d'une part que notre texte est constitué d'un ensemble de leçons, et obéit ainsi à une visée pédagogique, mais d'autre part qu'il ne s'y réduit pas, le cours étant suffisamment travaillé pour que Cramer puisse envisager sa publication.

Charles Bonnet indique de plus dans une lettre à Jean Senebier du 16 mai 1786 le nom de la destinataire du cours, Marie Charlotte Boissier-Lullin, nièce de $\mathrm{Mr}$ de Lubières, lequel prenait en notes le cours. Ceci permet à Jean-Daniel Candaux de situer la date probable où ces leçons furent prononcées à la fin de l'année 1744 ou au début de l'année $1745^{8}$.

\subsection{DESCRIPTION MATÉRIELLE}

Le manuscrit autographe est perdu. La Bibliothèque Publique et Universitaire de Genève en possède cinq copies, dont une incomplète. Les quatre autres sont identiques et formés d'un texte écrit en français, distribué en 576 paragraphes numérotés, d'inégale longueur. Le cours est inachevé, le $\S 556$, qui introduit le dernier moment destiné à l'étude des sources de l'erreur en distinguant trois, la précipitation, les préjugés et les passions, alors que le texte s'achève au $\$ 576$ sur l'analyse des préjugés. (Deux copies possèdent une suite, composée des paragraphes 577 à 624, écrite par de Lubières luimême, et analysant cette troisième source d'erreurs que sont les passions).

La copie que Jean-Daniel Candaux a bien voulu me communiquer et sur laquelle j'ai travaillé est le «MS Trembley 34 ». Il s'agit d'un texte de 348 pages au format A4, écrites de façon très lisible et régulière, présentant très peu de ratures et entièrement rédigé. Le texte des paragraphes est généralement précédé d'un titre qui en indique le contenu. Ces indications permettent de supposer que le cours, d'abord pris sous la dictée, a ensuite été patiemment recopié.

\footnotetext{
${ }^{6}$ Charles Benjamin de Lubières, né à Berlin en 1714 , est le $7^{\mathrm{e}}$ et seul survivant d'une famille de huit enfants, dont le père, calviniste et gouverneur d'Orange, s'exila à Berlin, après que Louis XIV ait conquis la principauté d'Orange. Correspondant de Voltaire, il fréquenta l'Académie de Genève et y suivit le cours de Gabriel Cramer, alors qu'il n'avait que 15 ans, et son professeur 25. Celui-ci avait été nommé à la première chaire de mathématiques de l'Académie de Genève, en compagnie de Jean-Louis Calandrini (qui, à partir de de 1734 occupera celle de philosophie). C. B. de Lubières est l'auteur de l'Éloge historique de Cramer publié dans la Nouvelle bibliothèque germanique d'Amsterdam pour l'édition de 1752, année de la mort de Cramer à 48 ans.

${ }^{7}$ [Bonnet, 1948, p. 145].

${ }^{8}$ Il s'appuie pour cela sur le fait que le cours ne peut être postérieur à mai 1750, date du décès tragique de Marie-Charlotte, mais est postérieur à mars 1744, date de son mariage, puisque Charles Bonnet ne parle pas d'une demoiselle, mais d'une jeune dame. Enfin, si l'on admet que la maternité n'est pas une période très favorable à l'étude, on peut supposer que le cours précédât les trois maternités successives de MarieCharlotte de juillet 1745, décembre 1746 et mars 1748. Le cours étant inachevé, on peut alors faire l'hypothèse qu'il fut interrompu justement par la maternité.
} 


\section{LA PROGRESSION DE L'ANALYSE}

\subsection{STRUCTURE DU COURS DE LOGIQUE}

Le Cours ne possède pas de table des matières, mais il est possible, à la lecture du texte, d'en dégager la structure et la progression, lesquelles nous renseignent sur la méthode de Cramer et la façon dont il conçoit les différentes formes et opérations de connaissance, leurs relations, ainsi que leur importance relative (raison pour laquelle j'indique, dans la suite, les paragraphes et les pages des développements qu'il me semble possible de distinguer). Les 22 premiers paragraphes constituent une introduction au cours, qui en définit l'objet et les parties. La logique est tout d'abord définie de manière traditionnelle comme «art de faire un bon usage de la raison » en vue de la connaissance de la vérité $(\S 1)$, cet art se réalisant soit sous la forme d'une logique naturelle, lorsque ses règles sont appliquées de manière spontanée et aveugle, soit sous celle de la logique artificielle, dont le but est de faire connaître distinctement ces règles et les principes qui les fondent. Cette dernière se compose de deux parties, comme l'indique Cramer lui-même, la première, "préliminaire », ayant pour objet d'expliquer ce que sont la raison d'abord, la vérité ensuite, la seconde, «principale qui montre l'usage qu'on doit faire de la raison pour parvenir à la connaissance de la vérité » (\$18). La première partie, définissant la vérité comme «conformité de nos pensées avec la nature des choses » (\$19), et la raison par l'ensemble des « opérations de l'esprit qui peuvent nous conduire à la vérité ou à l'erreur » $(\$ 20)$, prépare l'exposé du corps même de la logique artificielle, constitué par l'énoncé et l'étude des règles à mettre en œuvre dans la recherche de la vérité.

La première partie, intitulée «La raison » (\$§ 23 à 332, p. 3-190) est formée de trois sections, correspondant aux trois opérations élémentaires de l'esprit: 1) la formation des idées ( $\S \S 23$ à 246, p. 3-137), 2) leur relation dans des jugements ( $\S 247$ à 285 , p. 137-158), 3) l'enchaînement de ces jugements dans des raisonnements ( $\$ \S 286$ à 332, p. 158-190), ainsi que le note Cramer, résumant le mouvement de cette première partie au début du $\S 333$ :

\section{jusqu'ici nous avons vu comment l'esprit humain se forme des idées, les compare pour en porter des jugements et enchaîner ces jugements pour déduire les uns des autres.}

La seconde partie, intitulée «De la méthode » (\$\$ 333 à 576, p. 191-348), est formée d'une seule section, puisque, comme l'indique Cramer, elle a pour fonction d'exposer l'art de faire usage des trois opérations précédentes ${ }^{9}$. Il est cependant possible de l'organiser selon les indications que fournit le texte. Le $\S 548$, notamment, faisant suite à l'étude de la connaissance probable, débute par ces mots : « Mais en voilà bien assez sur cette matière et sur la probabilité en général. Et comme nous avons passé de la vérité à la simple vraisemblance, cette gradation nous conduit naturellement à quelques réflexions sur l'ignorance et ensuite sur l'erreur». Cette seconde partie se déploie donc selon quatre moments, le premier, consacré à la vérité ( $\$ \S 333$ à 447 , p. 191-260), en explore 1) les «formes » ordonnées par la distinction des vérités

\footnotetext{
${ }^{9}$ On aperçoit ici à la fois la relation du Cours de logique de Cramer à la Logique dite de Port-Royal et la distance qui les sépare. Quand Arnauld et Nicole, distinguant quatre opérations principales de l'esprit, à savoir concevoir, juger, raisonner et ordonner, organisent leur exposé en quatre parties consacrées successivement aux idées, aux jugements, aux raisonnements et enfin à la méthode, Cramer, quant à lui, regroupe les trois premiers moments, correspondant aux trois opérations fondamentales de la raison, au sein de la première partie, servant de propédeutique à l'étude de la logique elle-même que constitue l'exposé de la méthode comme art d'user de sa raison.
} 
métaphysique, logique et morale, 2) les «sources », reposant sur la distinction des vérités intellectuelles et des vérités sensibles, ce qui conduit d'abord à l'étude de la connaissance intellectuelle, donc de l'évidence ( $\$ \$ 345$ à 358, p. 195-205), ensuite à celle de la connaissance sensible, et donc de la connaissance empirique ( $\$ \S 359$ à 447, p. 205-260). Le second moment a pour objet le vraisemblable, donc la connaissance probable ( $\$ 448$ à 547, p. $260-327)$. Le troisième porte sur les causes de l'ignorance $(\S \S 548-555$, p. $327-333)$ et le quatrième sur les sources de l'erreur ( $\$ \S 556$ à 576, p. 333-348). Chacun de ces quatre moments se développe selon un même mouvement, prenant successivement pour objet la définition de son objet, la variété de ses formes, enfin les sources d'où il provient. Il faut remarquer cependant que l'importance respective accordée à chacun de ses trois objets est différente selon les moments, et que la notion de «source de connaissance » reçoit une signification très lâche. Ceci est particulièrement vrai du développement portant sur la connaissance probable, dont la progression ne suit que grossièrement ce schéma.

\subsection{STRUCTURE DU TEXTE CONSACRÉ À LA CONNAISSANCE PROBABLE (§§ 448 à 547)}

L'étude de la connaissance probable, si on accepte d'y inclure la construction de sa définition à partir de la notion de certitude, occupe un volume à peu près équivalent à celui de la vérité, puisqu'elle débute au $\S 448$ pour s'achever au $§ 547$ (p. 260-327). Le texte peut être ordonné plus précisément en trois moments principaux.

Le premier moment de l'analyse vise à mettre en place les définitions nécessaires à l'étude. Ce processus définitionnel s'effectue en deux temps. Le premier, servant de transition avec le moment précédent, conduit à définir la connaissance probable comme connaissance intermédiaire entre la certitude de la vérité et la certitude de la fausseté, à partir de l'analyse des notions de certitude et d'incertitude ( $\S 448$ à 454, p. 260-262). Le second pose les définitions des concepts de probabilité, de vraisemblance et de certitude morale, délimitant ainsi le champ de l'incertain ( $\$ 455$ à 459, p. 262-264).

Le second moment n'examine pas les différentes formes de probabilité, mais présente «quelques règles ou principes de bon sens », reprises en fait de l'Ars conjectandi de Jacques Bernoulli ( $\$ 460$ à 472, p. 264-274).

Enfin, un troisième moment, consacré aux « sources » de la probabilité, se déploie en trois volets; le premier $^{10}$ s'étend des $\$ \S 473$ à 480 (p. 274-280) et concerne la distinction entre probabilité a priori et probabilité a posteriori, que Cramer désigne pour sa part comme probabilité «prise de la nature même de la chose et des causes qui la produisent » et probabilité «prise de l'expérience du passé ». Le second volet, dont Cramer s'en tient à indiquer qu'il traite des «autres sources de probabilité » ou « principes les plus communs $»^{11}$ (\$§ 481 à 547, p. 280-327), prend successivement pour objet «l'égale possibilité de plusieurs événements [\$\$ 481-488], la connaissance des causes [ $\$ \$ 489-490]$, le témoignage [\$\$ 491-514], l'analogie [\$\$ 515-535] et les hypothèses [\$§ 536-547] ».

\footnotetext{
${ }^{10}$ Cours de Logique, $\S 473$ : «On pourrait multiplier sans doute ces règles et ces axiomes. Un peu de commerce avec des personnes intelligentes dans les affaires du monde en apprendra plus qu'il ne convient d'en mettre dans une Logique. Venons présentement aux sources de la probabilité ». Dans toutes les citations du texte, nous avons modernisé l'orthographe et la ponctuation afin d'en faciliter la lecture, dans la mesure où cela n'entraîne aucune ambiguïté.

${ }^{11}$ Ibid., $\$ 481$.
} 


\section{LA CONSTRUCTION DU CONCEPT DE PROBABILITÉ}

L'élaboration du concept de probabilité prend appui sur la définition de la certitude comme «connaissance évidente de la vérité ou de la fausseté d'une proposition» (§ 448), l'incertitude résultant a contrario du défaut de cette connaissance évidente. La vérité d'une proposition est elle-même définie (\$ 450) par la « convenance du sujet et de l'attribut » de cette proposition. Cette convenance est assurée si toutes les conditions qui la rendent possible sont réunies, ce qui conduit à distinguer trois cas :

- la proposition est connue comme vraie avec certitude, si l'on sait que toutes les conditions nécessaires sont satisfaites ( $\$ 451)$,

- elle est connue comme fausse avec certitude, si l'on sait qu'il manque une de ces conditions ( $\$ 452)$,

- elle est probable, si l'on ignore si toutes les conditions nécessaires à sa vérité sont réunies, et elle possède d'autant plus de vraisemblance que nous connaissons plus de conditions de sa vérité :

Mais quand il arrive, ce qui n'est que trop ordinaire que nous ne savons pas sûrement si toutes les conditions requises pour nous assurer de quelque vérité ont lieu, alors cette vérité n'est que probable; elle a d'autant plus de vraisemblance que nous voyons plus de conditions requises avoir certainement lieu ${ }^{12}$.

Cette dernière remarque a pour fonction d'installer une conception métrique de la probabilité : «C'est [donc] le nombre de ces conditions, poursuit Cramer, qui forme les degrés de probabilité ». Et cette représentation métrique vient à son tour autoriser la constitution de la connaissance probable en calcul de probabilités.

Gabriel Cramer achève alors ce premier moment en définissant le statut du texte. Il indique en effet : 1) que la connaissance probable, s'effectuant par degrés, est susceptible d'un traitement mathématique, 2) que la logique, distincte des mathématiques, laisse le calcul algébrique hors de son domaine, mais que 3) en tant qu'art d'utiliser sa raison dans la connaissance, elle en définit les principes et en établit les fondements :

Les mathématiciens qui veulent mêler leurs calculs partout n'ont pas laissé échapper cette matière. Ils se sont avisés d'employer l'algèbre pour supputer ces degrés, et quoique la logique n'ait rien à faire avec ces calculs, il est pourtant de son ressort d'en démontrer au moins les principes et les fondements, et d'en saisir la théorie d'une vue au moins générale ${ }^{13}$.

Ce premier moment débouche alors sur la définition rigoureuse de la probabilité, donnée au $§ 455$ :

Puisque la certitude entière naît de l'assurance que l'on a de l'existence de toutes les conditions ${ }^{14}$ requises pour certaines vérités, et la probabilité de la connaissance qu'on a de l'existence de quelques unes de ces conditions, on regarde la certitude comme un tout et la probabilité comme une partie. Le

\footnotetext{
${ }^{12}$ Ibid., $\$ 453$.

${ }^{13}$ Ibid., $\$ 454$.

${ }^{14}$ Ces « conditions » étant, précise le $\S 450$, «exprimées ou sous-entendues : exprimées quand l'attribut ne convient pas au sujet par son essence, mais en vertu de quelque cause ou raison tirée de dehors, sousentendue, quand il lui convient par son essence, qui porte avec elle les conditions nécessaires pour qu'on puisse attribuer l'attribut au sujet».
} 
juste degré de probabilité d'une proposition sera donc exactement connu quand on pourra dire et prouver que cette probabilité monte à une demi certitude ou aux trois quart de la certitude entière, ou seulement au tiers de la certitude, etc.

Cette définition logique de la probabilité reprend implicitement celle donnée par Jacques Bernoulli au début du $1^{\text {er }}$ chapitre de la $4^{\mathrm{e}}$ partie de l'Ars conjectandi : "la probabilité est un degré de la certitude et en diffère comme la partie diffère du tout ${ }^{15}$. Et Cramer reprend dans la suite la même démarche que celle suivie par Bernoulli, consistant à placer en regard de cette définition, la représentation commune - «l'usage ordinaire » dit Cramer - des différents degrés de probabilité, distinguant la certitude morale, le vraisemblable ${ }^{16}$ et $l^{\prime}$ 'incertain ${ }^{17}$. Cramer maintient donc, à côté de la représentation numérique de la probabilité, une détermination qualitative, héritée de la tradition, détermination qui s'exprimait déjà au $\S 352$, indiquant que les propositions sont dites probables «quand nous avons des preuves qui nous portent fortement à en croire la vérité ou la fausseté, quoiqu'elles ne suffisent pas pour mettre l'esprit dans cette situation qui ne laisse plus rien à désirer pour être entièrement certain ».

Ceci conduit alors à admettre deux structurations différentes du champ du probable. Dans le premier cas, le probable correspond à une région homogène allant du certainement faux au certainement vrai, chaque degré de probabilité pouvant s'exprimer par une valeur comprise entre 0 et 1 . Dans le second, il ne correspond qu'aux valeurs supérieures à $\frac{1}{2}$, la demi-certitude séparant le domaine du probable de celui du douteux.

On peut représenter cette double structuration en construisant le tableau suivant :

\begin{tabular}{|c|c|c|}
\hline & Probabilité qualitative & Probabilité mathématique \\
\hline \multicolumn{3}{|l|}{ Certainement vrai } \\
\hline \multicolumn{3}{|l|}{ Moralement certain } \\
\hline 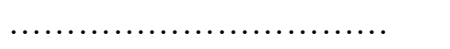 & probable & \\
\hline \multicolumn{3}{|l|}{ Vraisemblable } \\
\hline 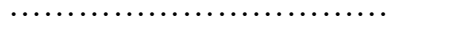 & & \\
\hline Incertain $=$ demi - certitude & & probable \\
\hline 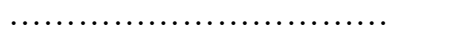 & & \\
\hline 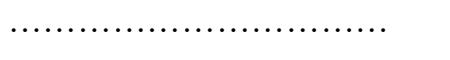 & douteux & \\
\hline n.w. & & \\
\hline Certitude morale de la fausseté & & \\
\hline Certainement faux & & \\
\hline
\end{tabular}

À la différence de Bernoulli, Gabriel Cramer se situe exclusivement sur le plan épistémique, et fait donc l'économie des définitions des concepts de nécessité, contingence, chance et accident, par lesquelles le premier achevait son chapitre

\footnotetext{
15 [Bernoulli, 1713, p. 211 ; Meusnier, 1987, p. 16].

${ }^{16}$ G. Cramer, Cours de Logique, $\S 456$ : «On appelle dans l'usage ordinaire probable, ce qui a plus d'une demi certitude ; vraisemblable, ce qui la surpasse considérablement ; et moralement certain ce qui approche beaucoup d'une certitude entière ».

17 Ibid., § 457 : «Une demi certitude forme l'incertain proprement dit où l'esprit trouvant de part et d'autre les raisons égales ne sait quel jugement porter, quel parti prendre ».
} 
inaugural. Il convient de noter à cet égard que, conformément à la définition de la probabilité en termes de degré de certitude, la probabilité ne mesure pas la possibilité de réalisation de l'événement lui-même, mais la vraisemblance des propositions ou jugements qui l'énoncent; la logique ayant pour tâche ici de nous permettre, autant qu'il est possible, «d'assigner à chaque proposition son juste degré de vraisemblance, et d'y proportionner son assentiment ${ }^{18}$. Il reprend alors le second chapitre de l'Ars conjectandi, dans lequel, après l'analyse définitionnelle précédente, Jacques Bernoulli faisait précéder l'étude proprement dite de l'art de conjecturer, de l'exposé de neuf règles préalables ou axiomes, définissant les conditions de son usage :

\begin{abstract}
Avant d'accéder de plus près à notre objet, où on montrera comment il convient d'utiliser ces arguments des conjectures pour mesurer les probabilités, je veux exposer d'abord quelques règles ou axiomes généraux, que la simple raison dicte à tout homme sain d'esprit et que les plus sages observent constamment dans la vie civile.
\end{abstract}

écrit Bernoulli ${ }^{19}$. À quoi répond le $§ 460$ du Cours de Logique de Gabriel Cramer :

Avant que d'examiner les sources vraies et fausses de la probabilité, il ne sera pas hors de propos d'avancer quelques règles ou principes de bon sens, et qui sont régulièrement observés par les personnes sages et prudentes.

Et Cramer reproduit presque littéralement ${ }^{20}$, et jusque dans les analyses d'exemples, ces neuf règles générales formant pour Jacques Bernoulli les réquisits de toute analyse probabiliste ; ainsi celle prescrivant de ne pas se contenter de probabilités, là où l'on peut atteindre la certitude, ou de prendre en compte l'ensemble des arguments qui peuvent soutenir la probabilité d'une proposition, comme aussi celle de sa contradictoire, etc. La seule originalité de Gabriel Cramer par rapport au texte de Bernoulli, outre l'étendue des développements, est d'adjoindre un dixième principe, ne figurant pas explicitement dans l'exposé de Bernoulli, constitué par une forme décisionnelle, et indéterminée, de la règle de l'espérance mathématique :

Lorsqu'il est question de se déterminer à agir, il ne faut pas seulement considérer la probabilité du succès, mais il faut encore peser la grandeur du bien ou du mal qu'on peut attendre en prenant un parti, ou en prenant le contraire, ou en restant dans l'incertain ${ }^{21}$.

\title{
4. LA PROBABILITÉ DES TÉMOIGNAGES
}

Dans la mesure où notre perception directe est limitée, et grandement, par nos conditions spatio-temporelles d'existence, nous sommes contraints de faire un usage fréquent de la connaissance par témoignage, et la probabilité des témoignages est donc un chapitre essentiel de la logique probabiliste ${ }^{22}$. Cramer commence par en définir

\footnotetext{
${ }^{18}$ Ibid., $\$ 470$.

${ }^{19}$ [Bernoulli, 1713, p. 214 ; Meusnier, 1987, p. 22].

${ }^{20}$ Cramer ne mentionne cependant pas le nom de Bernoulli ici, ne faisant référence à « feu M. Bernoulli, l'homme du monde qui entendait le mieux ces calculs » qu'au $\S 477$, à propos des probabilités obtenues par « réitération des expériences ».

${ }^{21}$ G. Cramer, Cours de Logique, $\$ 471$.

${ }^{22}$ Elle fait ultérieurement l'objet de développements notables au XVIII ${ }^{\mathrm{e}}$ siècle, de la part principalement de Lambert aux $\$ \S 230$ à 248 de la $5^{\mathrm{e}}$ section de son Nouvel Organon [Lambert, 1764 ; in Fanfalone p. 168-181], de Hume [1748, section X] et de Condorcet dans la première partie de l'Essai [Condorcet, 1785] et dans les $5^{\mathrm{e}}$ et $6^{\mathrm{e}}$ parties du Mémoire sur le calcul des probabilités [Condorcet, 1786-1787] ; en attendant, au siècle suivant, les analyses de Laplace [1812, p. 455-470] et [1814, in 1986, p. 118-131],
} 
l'objet, à savoir «déterminer le degré d'assentiment qu'on doit donner au témoignage $»^{23}$, et assigner son statut épistémologique : l'analyse sera réduite à la mise en place de « quelques règles » permettant d'en donner une représentation approchée eu égard, note Cramer, à la difficulté d'atteindre en ce domaine une «détermination précise ».

Ce degré d'assentiment dépend de deux facteurs, que sont d'une part le contenu même de la proposition déterminant le «degré de vraisemblance intrinsèque ${ }^{24} \mathrm{du}$ témoignage, de l'autre l'autorité des témoins déterminant son degré de vraisemblance extrinsèque ${ }^{25}$.

L'analyse du degré de vraisemblance intrinsèque du témoignage est constituée par la détermination de la possibilité ou impossibilité de l'événement, dans la mesure où, indique Cramer, dire d'une chose qu'elle est possible, c'est dire que rien dans sa nature ne s'oppose à son existence. Autrement dit, l'événement décrit par le témoignage est possible s'il n'y a pas de raison intrinsèque de le rejeter, cette impossibilité étant susceptible de recevoir trois formes :

- impossibilité absolue, ou métaphysique, ou encore géométrique, consistant à affirmer et nier simultanément le même contenu dans la même proposition,

- impossibilité physique, tenant à l'incompatibilité entre le fait considéré et les lois naturelles,

- impossibilité morale, résultant de l'opposition entre les actes et les qualités morales attribués à un sujet.

Du coup, l'analyse de la vraisemblance intrinsèque du témoignage ne fait pas l'objet d'un traitement probabiliste ${ }^{26}$, se réduisant à présenter les moyens de s'assurer que l'événement n'est pas impossible, puis à indiquer que le témoignage, « selon qu'il s'accorde mieux ou moins bien avec les faits connus, [...] a plus ou moins de vraisemblance $»^{27}$, mais sans chercher à préciser davantage la nature de cette relation.

Lacroix [1816, $\left.\mathrm{n}^{\text {os }} 140-150\right]$ qui en propose un historique, Poisson [1837, $\mathrm{n}^{\text {os }} 36-40$ ] et Cournot [1843, $\$ \S 224-225]$. Pour une analyse de la probabilité des témoignages au XVIII ${ }^{\mathrm{e}}$ siècle, notamment chez Condorcet, Laplace et Hume, on peut consulter [Zabell, 1988].

${ }^{23}$ G. Cramer, Cours de Logique, $\$ 491$.

${ }^{24}$ Ibid., $\$ 500$.

${ }^{25}$ Cette distinction, issue de la tradition logique et juridique, est vraisemblablement reprise par Cramer via l'Ars conjectandi, dans lequel Bernoulli définit les «arguments extrinsèques », comme ceux qui sont «issus de l'autorité et du témoignage des hommes» (op. cit., p. 22) - de la Logique de Port-Royal invitant, pour juger de la vérité d'un événement, à tenir compte des circonstances aussi bien intérieures qu'extérieures, en entendant par «circonstances intérieures celles qui appartiennent au fait même, et extérieures celles qui regardent les personnes par le témoignage desquelles nous sommes portés à le croire » [Arnauld et Nicole, 1662-1683, IV ${ }^{\mathrm{e}}$ partie, ch. XIII, p. 340].

${ }^{26}$ Ainsi que me l'a précisé Bernard Bru, si Condorcet reprendra l'idée de vraisemblance intrinsèque, qu'il appellera la «probabilité propre du fait», son originalité sera de lier ensemble les vraisemblances intrinsèque et extrinsèque pour calculer la probabilité de la vérité du fait. On peut, sur ce point, consulter la cinquième partie du Mémoire sur le calcul des probabilités de Condorcet, et les notes qui l'accompagnent, dans [Condorcet, 1994, p. 431-448], édité par Bernard Bru et Pierre Crépel, ainsi que son mémoire inachevé «Sur l'application du calcul à la probabilité des faits », donné dans le même ouvrage aux pages 557 à 573.

${ }^{27}$ « Ajoutons à ces degrés de vraisemblance tirés de la nature intrinsèque de la chose, ceux qu'elle tire de son accord avec d'autres vérités connues et établies d'ailleurs », écrit Cramer dans ce même $\$ 499$. L'analyse de la vraisemblance intrinsèque inclut donc elle-même deux temps, le premier consacré à l'étude des conditions internes de possibilité de l'événement, l'autre à celle de son accord avec les autres faits connus, si bien que la vraisemblance du témoignage dépend en fait de trois facteurs et non deux. Ceci sera explicite chez Lambert, qui précisera au $\$ 233$ de son Nouvel Organon que « le jugement relatif 
L'analyse du degré de vraisemblance extrinsèque du témoignage détermine sa validité $^{28}$. Elle dépend de deux paramètres : 1) le nombre des témoins, 2) la confiance dont ils sont susceptibles. L'analyse menée par Cramer de la validité du témoignage consistera alors d'une part à indiquer les différents éléments permettant d'estimer la confiance qu'on peut accorder à un témoin, de l'autre, et surtout, à énoncer deux lois de variation du degré de validité du témoignage en fonction du nombre des témoins, et donc à définir deux modes de calcul de ce degré de validité, le cœur de l'analyse consacrée à la probabilité des témoignages étant constitué par la mise en place de ces deux lois, à savoir :

1) la loi de croissance de la probabilité du témoignage en fonction du nombre de témoins directs,

2) la loi d'affaiblissement de la probabilité du témoignage en fonction du nombre de témoins indirects, c'est-à-dire par ouï-dire.

L'établissement de ces deux lois mobilise la même démarche, dont la progression est strictement observée, comme on peut le voir en considérant successivement leur exposition, se déployant en cinq temps.

\subsection{LOI DE CROISSANCE DE LA PROBABILITÉ DES TÉMOIGNAGES SIMULTANÉS}

Le premier temps de l'analyse se réduit à énoncer la loi sous une forme indéterminée, «plus il y a de témoins, plus le degré de vraisemblance augmente ${ }^{29}$, énoncé qui est donné pour évident : «pour ce qui est du nombre des témoins, il n'est personne qui ne sente que leur témoignage est d'autant plus croyable qu'ils sont plus nombreux ».

Le second temps est constitué par la formulation du problème. La construction du problème consiste ici à montrer que la solution qui s'offre immédiatement pour mesurer la probabilité du témoignage conduit à une absurdité, ce qui exige d'élaborer une méthode adéquate. Cramer pose, en effet, qu'on aurait spontanément tendance à croire que la probabilité du témoignage croît en raison directe du nombre des témoins :

On se persuaderait même aisément qu'il augmente de vraisemblance en même proportion que leur nombre augmente, de sorte que deux témoins d'une égale confiance feront une probabilité deux fois plus forte qu'un seul, et que trois en feront une triple et ainsi de suite.

Et il montre sur un exemple que cette hypothèse est inadmissible, car contradictoire avec la définition de la probabilité. Si, dit-il, le premier témoin «me donne une vraisemblance qui soit les $\frac{9}{10}$ de la certitude », et si j'accorde au second

\footnotetext{
à l'exactitude et à l'authenticité d'une information est fondé : 1) en partie sur la recherche de la chose elle-même, si elle est racontée. Elle ne doit contenir rien de contradictoire et donc aussi rien qui ne soit métaphysiquement ou physiquement impossible ; 2) en partie sur la comparaison de l'information avec les circonstances qu'elle présuppose et avec les conséquences que la chose, dans le cas où elle est vraie, doit avoir ; 3) sur l'examen de la crédibilité du témoin, de celui qui affirme ou de celui qui répète, à savoir si celui-ci peut dire la vérité et s'il veut la dire », [Lambert, op. cit, p. 172].

${ }^{28}$ Cramer hérite ici vraisemblablement de la théorie des témoignages simultanés de George Hooper, publiée d'abord anonymement en 1699 dans les Philosophical Transactions of the Royal Society (dont [Lacroix, 1816, p. 262] note qu'elle est ensuite reprise par [Bicquilley, 1783]), et des Principes mathématiques de théologie chrétienne de John Craig publiés à Londres la même année [Craig, 1699].

${ }^{29}$ G. Cramer, Cours de Logique, $\S 500$ bis. Je désigne par « 500 bis » un paragraphe qui, situé entre les $\S 500$ et 501 , est dépourvu de numéro d'ordre.
} 
témoin le même degré de confiance, il faudrait alors admettre que le second témoignage ajoute sa probabilité à celle du premier, si bien que la conjonction des deux témoignages attribuerait à la proposition $\frac{18}{10}$ de certitude, donc une probabilité $p>1$.

Le troisième temps donne l'énoncé de la solution, sur un exemple numérique :

Je dis donc, écrit Cramer, que le second témoin augmente la probabilité du premier qui était $\frac{9}{10}$ et la pousse à $\frac{99}{100}$, qu'un $3^{e}$ la pousse à $\frac{999}{1000}$, un $4^{e} \grave{a}$ $\frac{9999}{10000}$ et ainsi de suite approchant toujours plus de la certitude absolue, mais néanmoins sans y jamais arriver entièrement.

Le quatrième temps de l'analyse, introduit par la formule «mais voici la preuve de ce que je dis », est consacré à la démonstration de la solution précédemment indiquée. Cette démonstration est double, faisant appel à deux méthodes différentes.

1) Démonstration par la règle de la «probabilité composée », laquelle, héritée explicitement de 's Gravesande (cf. ['s Gravesande, 1737, § 641]), est définie comme « une probabilité entée sur une autre probabilité, ou la probabilité d'un événement qui ne peut arriver qu'au cas qu'un autre événement lui-même simplement probable arrive ${ }^{30}$. Cramer montre que si mon degré de confiance affecté au premier témoin est de $\frac{9}{10}$ de certitude, la probabilité qu'il me trompe est de $\frac{1}{10}$, et la probabilité que le second témoin me trompe également, donc confirme cette tromperie, est «le dixième d'un dixième, ou un centième ». La probabilité du témoignage est, par conséquent, de $\frac{99}{100}$.

2) Démonstration en termes de limite : Cramer définit la certitude comme la limite vers laquelle font tendre les témoignages. On mesure alors la probabilité du témoignage par la distance qu'il fait parcourir à ma croyance vers cette limite. Cramer pose ainsi que si le premier témoin est crédible avec $\frac{9}{10}$ de certitude, il nous fait parcourir les $\frac{9}{10}$ de la distance qui nous sépare de la certitude. Le second nous fait parcourir les $\frac{9}{10}$ du dixième restant, et ainsi de suite. Autrement dit, il n'ajoute pas à la validité du témoignage la même probabilité que le précédent, mais la même fraction de sa probabilitée ${ }^{31}$.

\footnotetext{
${ }^{30}$ Ibid., $\S 485$.

31 «Qu'on se représente la certitude entière comme la borne d'une carrière que les divers témoins qui viennent à l'appui l'un de l'autre nous font parcourir. Le premier nous en approche d'une quantité qui a avec toute la lice, la même proportion que la force de son témoignage a avec la certitude entière. Si son assurance produit chez moi les $\frac{9}{10}$ de la certitude, je conçois que le $1^{\text {er }}$ témoin me fait faire les $\frac{9}{10}$ du chemin vers la certitude. Vient un $2^{\mathrm{e}}$ témoin aussi croyable, que fait-il de plus ? Il m'avance sur la dixième restante précisément autant que le $1^{\mathrm{er}}$ témoin $\mathrm{m}$ 'avait avancé sur l'espace total; comme le $1^{\mathrm{er}}$ m'avait déjà amené aux $\frac{9}{10}$ du chemin vers la certitude, celui-ci m'en approche encore des $\frac{9}{10}$ de cette dixième restante, de sorte qu'avec ces deux aides, j'ai fait les $\frac{99}{100}$ du tout », Ibid., § 501.
} 


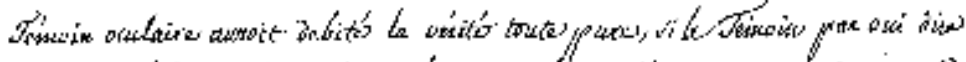

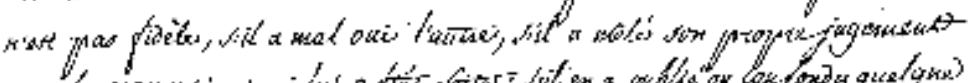

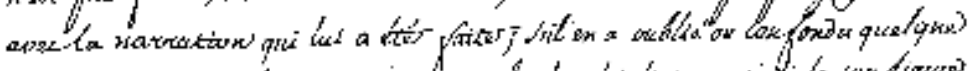

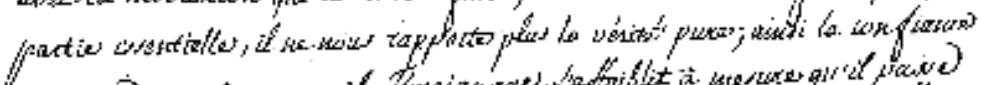

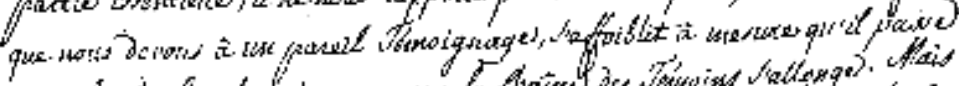

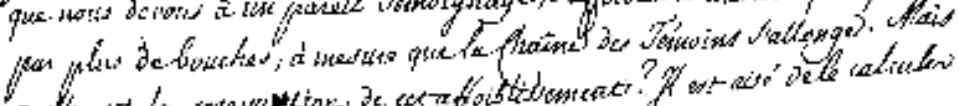

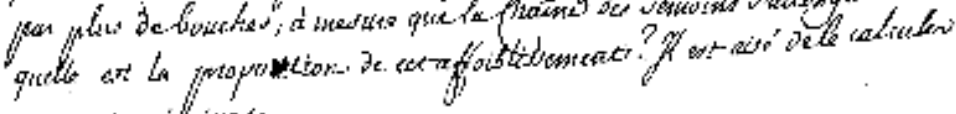

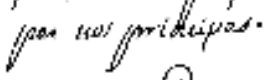

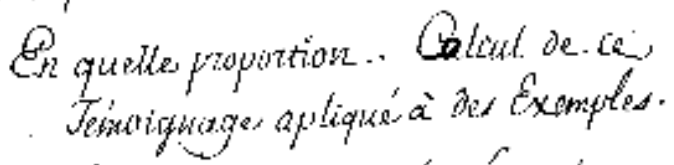

sot.

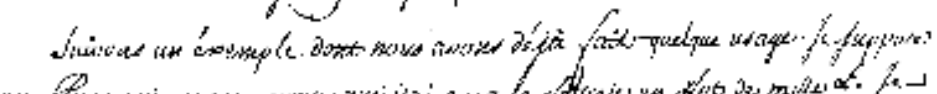

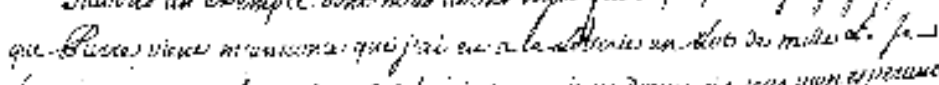

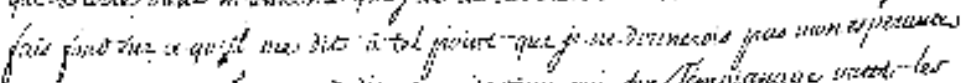

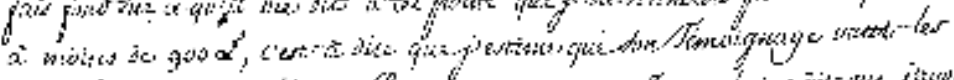

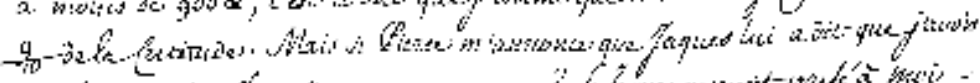
u w. 2 kn=

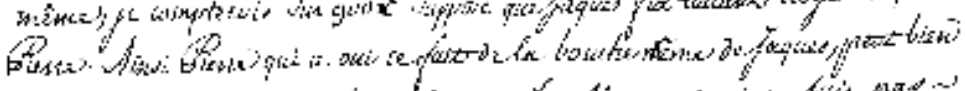

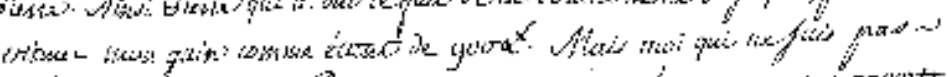

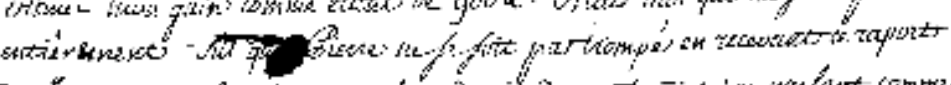

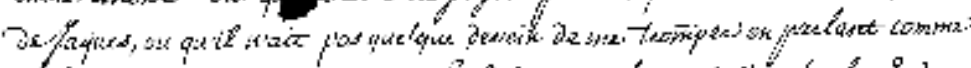

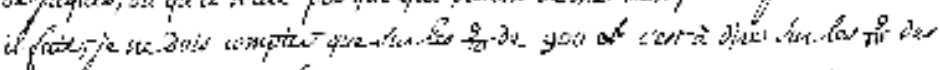

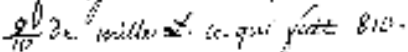

$\varepsilon_{\text {gr }}$ ast

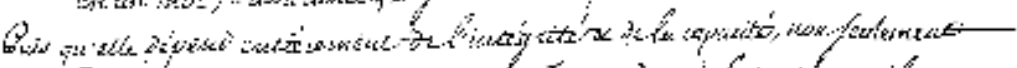

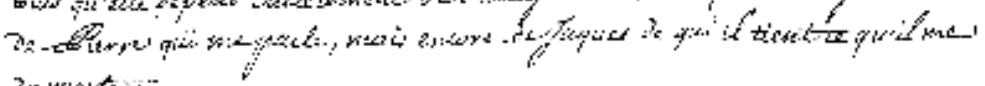
zerpost.

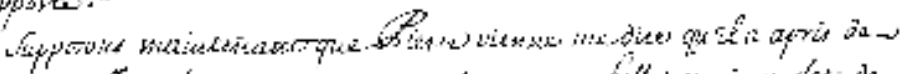

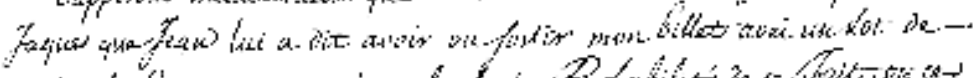

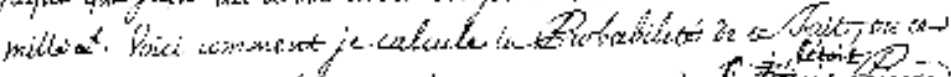

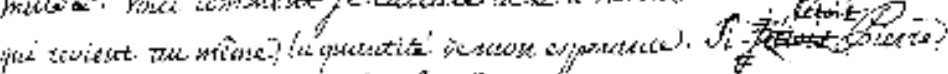

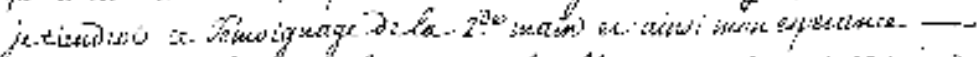

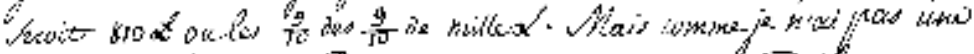

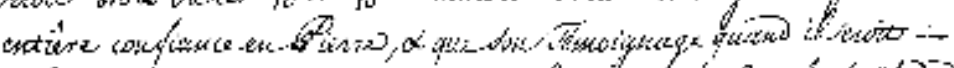

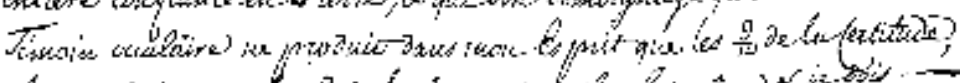

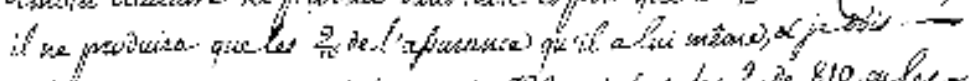

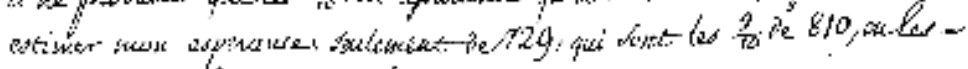
$\frac{9}{10}$ des $\frac{9}{1 ;}$ aet $\frac{9}{70}$ tow willo: 2 .

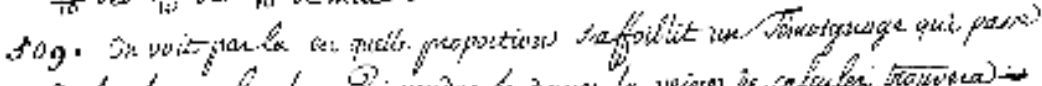

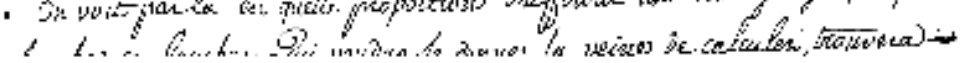




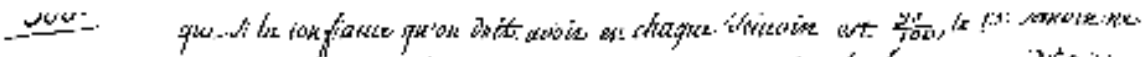

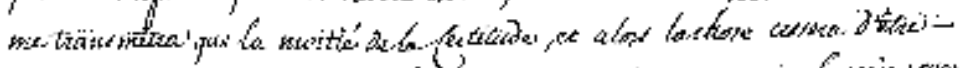

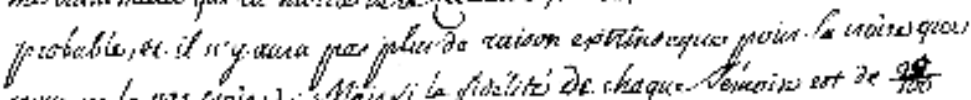

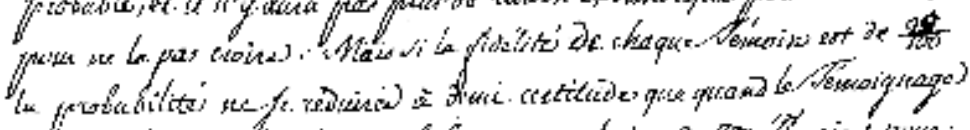

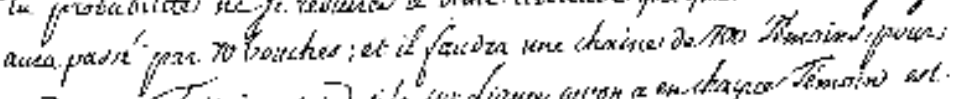

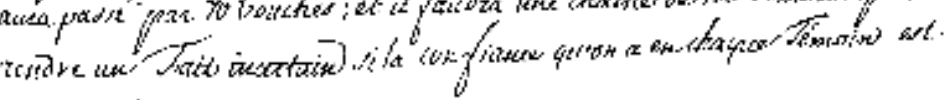

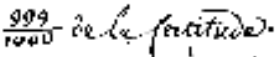

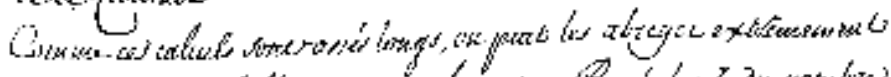

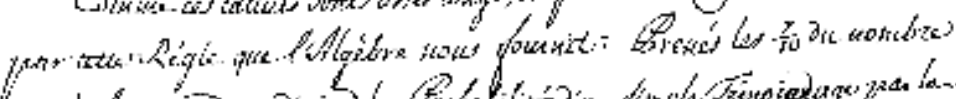

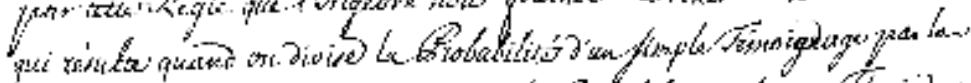

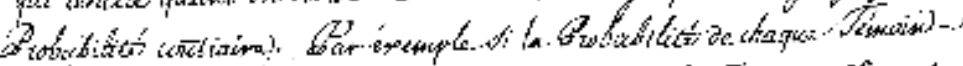

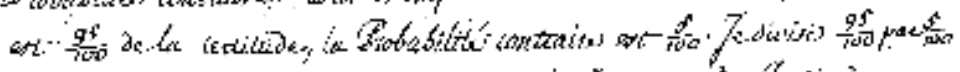

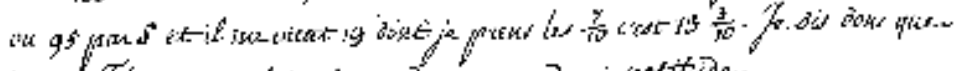

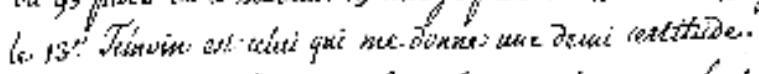

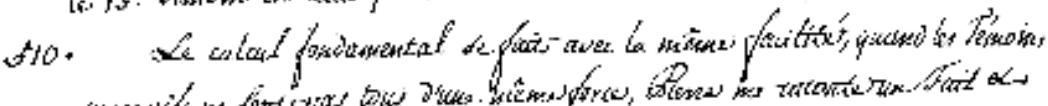

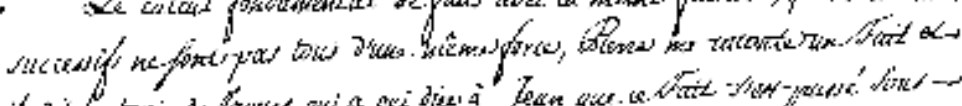

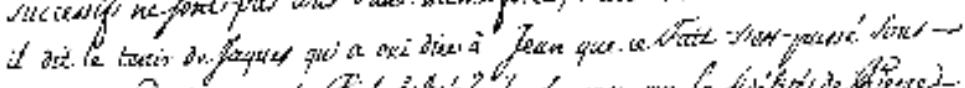

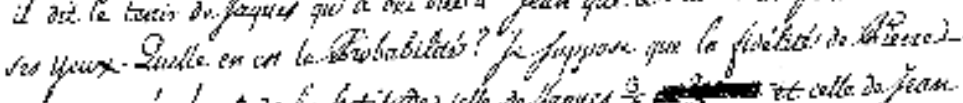

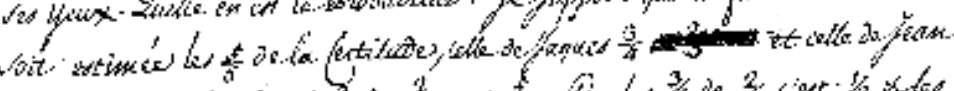

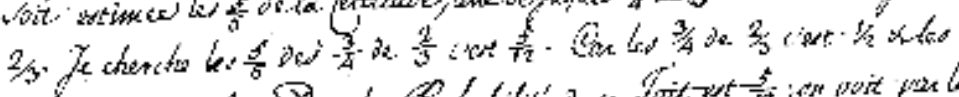

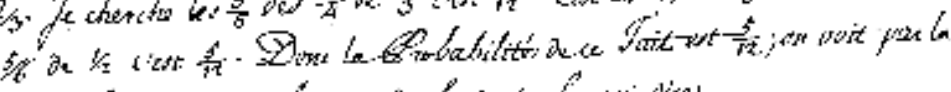

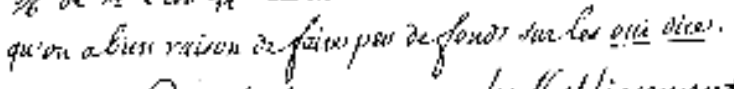

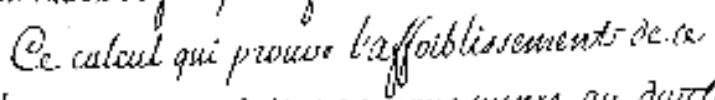

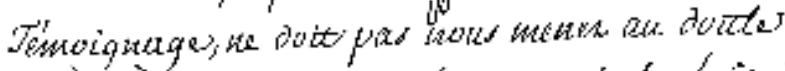

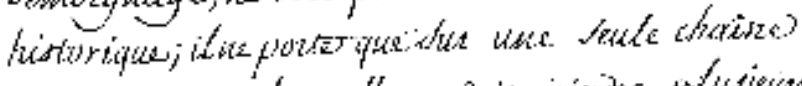

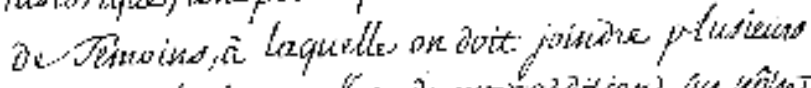

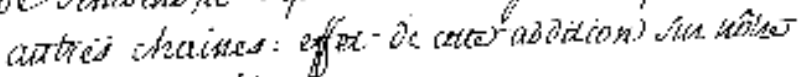
foi hilorique.

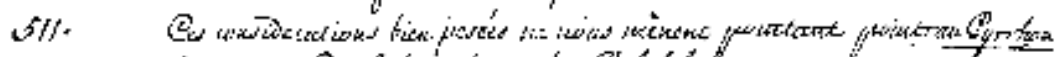

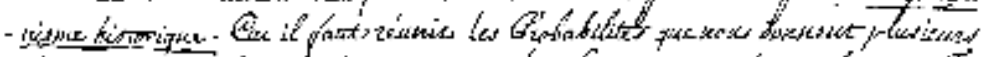

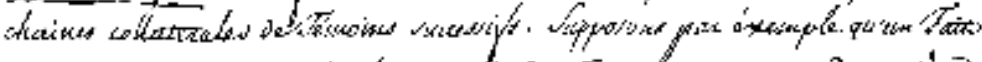

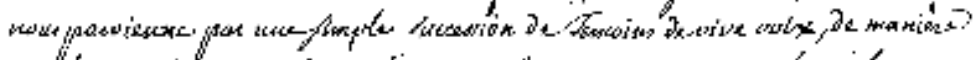

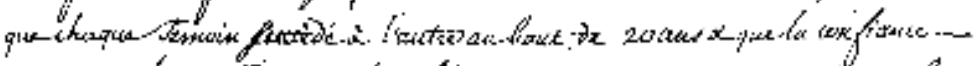
Pimrisece

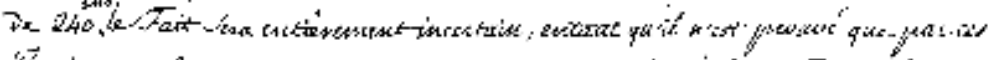

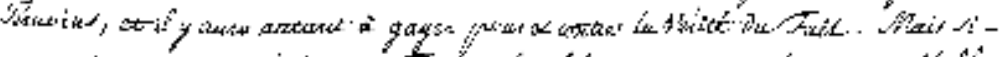

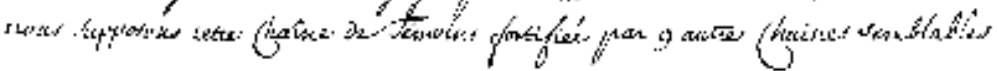


Enfin, le cinquième temps de l'analyse procède à l'assignation du caractère général de la méthode :

Cette méthode s'applique avec la même facilité à un nombre quelconque de témoins (ou de preuves concourantes) soit qu'on les suppose tous également croyables, soit qu'on leur assigne à chacun son degré particulier d'autorité, supposition plus conforme que la précédente à la nature des choses ${ }^{32}$.

Gabriel Cramer l'illustre en montrant comment, en affectant au second témoin un degré de confiance différent du premier, il fait parcourir à la croyance une fraction correspondante de la distance restante. Et il ajoute en corollaire de cette affirmation de généralité de la méthode, qu'elle est indépendante de l'ordre des arguments, donc de l'ordre de réception des témoignages.

\subsection{LOI D’AFFAIBLISSEMENT DE LA PROBABILITÉ DES TÉMOIGNAGES SUCCESSIFS ${ }^{33}$}

Comme précédemment, le premier temps de l'analyse est constitué par l'énoncé de la loi sous une forme indéterminée, que donne le titre du $§ 507$ :

\section{Observations sur les témoins par oui dire ; leur témoignage est moins croyable que celui d'un témoin oculaire. Pourquoi. Plus ce témoignage passe par différentes bouches, plus il s'affoiblit.}

Le second temps, consacré à la construction du problème, pose que la fiabilité du témoin indirect est plus faible que celle du témoin direct («il est bien clair qu'un témoin par oui dire, quoique d'ailleurs aussi honnête homme et aussi intelligent qu'un témoin oculaire, est pourtant moins croyable ${ }^{34}$ ), et ceci parce que les risques d'erreur sont accrus lors de la transmission du message. Cramer en déduit que le nombre des intermédiaires fait décroître la probabilité du témoignage, et la question se pose alors de savoir selon quelle proportion a lieu cet affaiblissement :

La confiance que nous devons à un pareil témoignage s'affoiblit à mesure qu'il passe par plus de bouches, à mesure que la chaîne des témoins s'allonge. Mais quelle est la proportion de cet affoiblissement?

Le troisième temps de l'analyse énonce la solution numérique du problème, en reprenant le même exemple que précédemment, celui d'un témoin dont le témoignage jouit d'un degré de vraisemblance égal à $\frac{9}{10}$ de certitude. Il montre que la probabilité de ce témoignage, que l'on suppose de même force que le précédent, est $\frac{9}{10}$ de $\frac{9}{10}$, donc une «probabilité de probabilité », puisque, dit-il, «elle dépend entièrement de l'intégrité et de la capacité non seulement de Pierre qui me parle, mais encore de

\footnotetext{
${ }^{32}$ Ibid.

${ }^{33}$ Énoncée par Craig dans ses Principia mathematica de 1699, elle est reprise sous forme littéraire par Locke au chapitre XVI du livre IV de son Essai philosophique concernant l'entendement humain ([Locke, 1700, rééd. 1972, p. 553-554]), à qui sans doute Cramer l'emprunte. Elle sera ultérieurement reprise dans cette tradition anglaise notamment par [Hume, 1739, 1. I, part. III, sec. 13] et [Bentham, 1827]. Sur Bentham, on peut consulter [Cléro, 2004].

${ }^{34}$ Ibid., $\$ 507$.
} 
Jacques de qui il tient ce qu'il me rapporte $»^{35}$. Et il applique ensuite cette solution numérique au cas où l'on considère trois témoins successifs.

Il en déduit, mais sans fournir le calcul correspondant, le nombre de témoins indirects à partir duquel le degré de validité du témoignage équivaut à une demicertitude, c'est-à-dire le seuil à partir duquel le témoignage jouit d'une probabilité égale à $\frac{1}{2}$; qualitativement, celui à partir duquel il passe du probable au douteux :

On voit par là en quelle proportion s'affaiblit un témoignage qui passe de bouche en bouche. Qui voudra se donner la peine de calculer, écrit-il, trouvera que si la confiance qu'on doit avoir en chaque témoin est $\frac{95}{100}$, le $13^{e}$ témoin ne me transmettra que la moitié de la certitude, et alors la chose cessera d'être probable, et il n'y aura pas plus de raison extrinsèque pour la croire que pour ne la pas croire ${ }^{36}$.

Le quatrième temps énonce la règle générale permettant d'obtenir le résultat numérique précédent. Elle prescrit de diviser par $\frac{7}{10}$ le rapport de la probabilité à la probabilité contraire, pour obtenir la valeur du seuil en deçà duquel le témoignage devient douteux, par conséquent pour obtenir le nombre de témoins indirects requis pour ce passage :

$$
\begin{aligned}
& \text { Prenez les } \frac{7}{10} \text { du nombre qui résulte quand on divise la probabilité d'un } \\
& \text { simple témoignage par la probabilité contraire. Par exemple, si la } \\
& \text { probabilité de chaque témoin est } \frac{95}{100} \text { de la certitude, la probabilité contraire } \\
& \text { est } \frac{5}{100} \text {. Je divise } \frac{95}{100} \text { par } \frac{5}{100} \text { ou } 95 \text { par } 5 \text { et il me vient } 19 \text { dont je prends les } \\
& \frac{7}{10} \text {. Je dis donc que le } 13^{e} \text { témoin est celui qui me donne une demi-certitude. }
\end{aligned}
$$

Comme c'était le cas lors de l'édification de la loi précédente, ce quatrième temps procède à une généralisation de la solution, mais cette fois sans en fournir la démonstration. Cette différence ne tient pas à un changement de méthode dans la construction de la solution, mais à des raisons pédagogiques, comme l'indique la formule par laquelle Cramer annonce ce quatrième temps, indiquant que «comme ces calculs sont assez longs, on peut les abréger extrêmement par cette règle que l'algèbre nous fournit ». En effet, la nature du texte, comme la personne à qui il s'adresse, ne permettent pas d'en passer par le calcul de logarithmes que nécessite la solution algébrique qui engendre la formule ici proposée.

Enfin, là encore, le cinquième temps de l'analyse a la charge de manifester le caractère général de la méthode. Cramer affirme ici que la méthode s'applique tout autant à des témoignages dont les degrés respectifs de validité sont différents (et non, évidemment, quel que soit le nombre de témoins, puisque cela est déjà contenu dans la construction de la solution), et l'illustre sur un exemple numérique. On peut remarquer que l'affirmation de la généralité de la méthode mobilise la même expression que

\footnotetext{
${ }^{35}$ Ibid., $\S 508$.

${ }^{36}$ Ibid., $\$ 509$.
} 
précédemment, la méthode s'appliquant, dit Cramer, «avec la même facilité » aux différents cas possibles.

Or, si l'on confronte le texte de Gabriel Cramer avec celui que donne l'Encyclopédie, force est de constater que cette rigueur démonstrative est compromise.

Ainsi, après avoir énoncé la règle générale permettant de déterminer le nombre de témoins indirects à partir duquel le témoignage vaut une demi-certitude, Cramer conclut l'ensemble de l'analyse du témoignage indirect en écrivant : «Le calcul fondamental se fait avec la même facilité quand les témoins successifs ne sont pas tous d'une même force », formule qui est suivie d'une application numérique montrant comment mesurer la probabilité du témoignage lorsque les témoins sont de force différente :

Pierre me raconte un fait et il dit le tenir de Jacques qui a oui dire à Jean que ce fait s'est passé sous ses yeux. Quelle en est la probabilité ? Je suppose que la fidélité de Pierre soit estimée les $\frac{5}{6}$ de la certitude, celle de Jacques $\frac{3}{4}$ et celle de Jean $\frac{2}{3}$. Je cherche les $\frac{5}{6}$ des $\frac{3}{4}$ de $\frac{2}{3}$, c'est $\frac{5}{12}$. Car les $\frac{3}{4}$ de $\frac{2}{3}$ c'est $\frac{1}{2}$, et les $\frac{5}{6}$ de $\frac{1}{2}$ c'est $\frac{5}{12}$. Donc la probabilité de ce fait est $\frac{5}{12}{ }^{37}$.

Or, le texte de l'Encyclopédie, après avoir repris l'exposé de la solution numérique du problème telle que la formule Cramer dans le troisième moment de son analyse, condense les quatrième et cinquième temps dans le passage suivant :

Ces calculs assez longs peuvent être abrégés par cette règle générale, dont l'algèbre simple nous fournit le résultat et la démonstration. Prenez les $\frac{7}{10} d u$ quotient de la division de la probabilité d'un simple témoin par la probabilité contraire, comme ici de $\frac{95}{100}$ par $\frac{5}{100}$, ou de 95 par 5, qui est 19, dont je prends les $\frac{7}{10}$, \& vous aurez le témoin qui vous laisse dans une demicertitude ; dans cet exemple c'est $13 \frac{3}{10}$, ce qui donne le treizième témoin. Il en sera de même si les témoins successifs sont supposés de force inégale ${ }^{38}$.

Cette dernière formule, allusive, semblant s'appliquer au calcul qui vient d'être proposé, rend l'ensemble sinon incompréhensible, du moins particulièrement obscur. Ainsi, contraint sans doute par des impératifs de volume, $\mathrm{Mr}$ de Lubières joue la brièveté contre l'intelligibilité.

\section{CONCLUSION}

Après lecture du texte, il apparaît que notre hypothèse initiale est satisfaite en un double sens. D'une part, d'un point de vue historique, le texte de Cramer illustre les difficultés qu'a rencontré le XVIII ${ }^{\mathrm{e}}$ siècle à penser de manière rigoureusement numérique la probabilité. Il s'offre, à cet égard, comme un bon témoin de l'état du développement de la pensée probabiliste dans la première moitié du XVIII ${ }^{\mathrm{e}}$ siècle. D'autre part, même s'il

\footnotetext{
${ }^{37}$ Ibid., $\S 5$.

${ }^{38}$ [Anonyme, 1765, p. 399ab] et [Anonyme, 1785, p. 648b].
} 
ne mobilise pas directement les ressources de l'algèbre, Cramer fait preuve dans l'édification de son cours d'une exigence de rigueur et de méthode qui se lit dans l'organisation très structurée et progressive qui commande l'exposition de son propos et la construction de son argumentation, assurant à son texte une clarté démonstrative qui ne se retrouve pas dans le résumé qu'en donne l'Encyclopédie ${ }^{39}$.

\section{BIBLIOGRAPHIE}

CRAMER G., Cours de logique, Genève, Bibliothèque Publique et Universitaire de Genève, MS Trembley 34, 1745 ?, 348 f.

CRAMER G., «Cours de logique (paragraphes 448-547)», reproduction partielle du MS Trembley 34 de la Bibliothèque Publique et Universitaire de Genève, par Th. Martin, in Journ@l électronique d'histoire des probabilités et des statistiques, www.jehps.net, vol. 2, $\mathrm{n}^{\circ} 1$, juin 2006, $6+89$ p.

Anonyme, «Probabilité », Encyclopédie ou Dictionnaire raisonné des sciences, des arts et des métiers, par une société de gens de Lettres [Diderot et d'Alembert éd.], tome treizième, reprod. de l'éd. de, Neufchastel, chez Samuel Faulche, 1765, p. 393b-400a ; reprod. électronique in Journ@l électronique d'histoire des probabilités et des statistiques, www.jehps.net, vol. 2, nº 1, juin 2006.

Anonyme, «Probabilité », Encyclopédie méthodique. Mathématiques, tome II, Paris, Panckoucke, 1785, reprod. Paris, ACL-éditions, 1987, p. 640b-649a; reprod. électronique inJourn@l électronique d'histoire des probabilités et des statistiques, www.jehps.net, vol. 2, $\mathrm{n}^{\circ}$ 1, juin 2006.

ARNAULD A., NICOLE P., [1683], La Logique ou l'Art de Penser..., édité par Pierre Clair et François Girbal, Paris, Presses Universitaires de France, 1965.

BENTHAM J., Rationale of judicial evidence, London, Hunt and Clarke, 1827.

BERNOULLI J., Ars conjectandi, Bâle, 1713. [Edition bilingue (latin - français) de la $4^{\mathrm{e}}$ partie dans Meusnier 1987].

BICQUILLEY C. F. (de), Du calcul des probabilités, Toul, Joseph Carez, 1783.

BONNET Ch., Mémoires autobiographiques, édités par Raymond Savioz, Paris, Vrin, 1948.

BUFFON G. L. Leclerc, (comte de), «Essai d'arithmétique morale», in Histoire naturelle, générale et particulière, servant de suite à l'Histoire naturelle de l'Homme, Supplément, tome quatrième, Paris, Imprimerie Royale, 1777.

CANDAUX J.-D., «Un auteur (et même deux) pour Idée, Induction, Probabilité: Monsieur de Lubières, encyclopédiste », Recherches sur Diderot et sur l'Encyclopédie, $\mathrm{n}^{\circ} 15$, oct. 1993, p. 71-96.

CLÉRO J.-P., Bentham et les probabilités. Esquisse d'une lecture du Rationale of judicial evidence, Paris, Les Cahiers du C.A.M.S., $\mathrm{n}^{\circ}$ 234, 2004.

CONDORCET N. (de), Essai sur l'application de l'analyse à la probabilité des décisions rendues à la pluralité des voix, Paris, 1785. [Réimpr. Chelsea, 1972].

CONDORCET N. (de), "Mémoire sur le calcul des probabilités », Mémoires de l'Académie royale des sciences, 1784-1787. [Réédité dans Condorcet 1994, p. 387-448].

\footnotetext{
39 On peut le vérifier aisément en confrontant le texte de Cramer à l'article «Probabilité » de l'Encyclopédie, reproduits au vol. 2, $\mathrm{n}^{\circ} 1 \mathrm{du}$ Journ@l électronique d'histoire des probabilités et des statistiques.
} 
CONDORCET N. (de), Sur l'application du calcul à la probabilité des faits, [réédité dans Condorcet, 1994, p. 557-573].

CONDORCET N. (de), Arithmétique politique. Textes rares ou inédits (1767-1789). [Edité et annoté par Bernard Bru et Pierre Crépel, Paris, Ined, 1994].

COURNOT A. A., Exposition de la théorie des chances et des probabilités, Paris, Hachette, 1843, [rééd. avec notes de Bernard Bru, Paris, Vrin, 1984].

CRAIG J., Theologiae christianae principia mathematica, London, 1699.

's GRAVESANDE W. J., Introduction à la philosophie, contenant la métaphysique et la logique, traduite du latin par Élie de Joncourt, Leide, J. et H. Verbook, 1737.

HANKS L., Buffon avant l'"Histoire Naturelle », Paris, Presses Universitaires de France, 1966.

HOOPER G., «A Calculation of the cridibility of human terstimony », Philosophical Transactions of the Royal Society, XXI, 1699, p. 359-365.

HUME D., A Treatise of human nature, London, 1739. [Traduction française par A. Leroy, Traité de la nature humaine, Paris, Aubier, 1946].

HUME D., An enquiry concerning human understanding, 1748. [Traduction française par A. Leroy, Enquête sur l'entendement humain, Paris, Garnier-Flammarion, 1983].

LACROIX S.-F., Traité élémentaire du calcul des probabilités, Paris, Courcier, 1816.

LAMBERT J.-H., Neues Organon..., Leipzig, Johann Wendler, 1764. [Traduction française de Gilbert Fanfalone, Nouvel Organon, 1764, traduction française, Paris, Vrin, 2002].

LAPLACE P.-S. (de), Théorie analytique des Probabilités, Paris, Courcier, 1812.

LAPLACE P.-S. (de) Essai philosophique sur les probabilités, Paris, Courcier, 1814, [rééd. avec notes de Bernard Bru, Paris, Christian Bourgeois, 1986.

LOCKE J., Essai philosophique concernant l'entendement humain, 1700. [Traduction française par Pierre Coste, Amsterdam, réédition de la $5^{\mathrm{e}}$ édition de 1755, Paris, Vrin, 1972].

MAYER J., «Diderot et le calcul des probabilités dans l'Encyclopédie », Revue d'histoire des sciences, tome XLIV, 1991, p. 375-391.

MEUSNIER N., Jacques Bernoulli et l'ars conjectandi, Université de Rouen Haute Normandie, IREM, 1987.

POISSON S.-D., Recherches sur la probabilité des jugements en matière criminelle et en matière civile, précédées des Règles générales du calcul des probabilités, Paris, Bachelier, 1837.

WEIL F., «La correspondance Buffon-Cramer », Revue d'histoire des sciences, t. XIV, $\mathrm{n}^{\circ} 2$, avril-juin 1961, p. 97-136.

ZABELL S. L., "The probabilistic analysis of testimony », Journal of Statistical Planning and Inference, vol. 20, $n^{\circ} 3,1988$, p. 327-354. 